\title{
TIM3 expression on TILs is associated with poor response to neoadjuvant chemotherapy in patients with locally advanced triple-negative breast cancer
}

Neslihan Cabioglu ${ }^{1 *}$ (D), Semen Onder ${ }^{2}$, Gizem Oner ${ }^{1,3,4}$, Hüseyin Karatay², Mustafa Tukenmez ${ }^{1}$, Mahmut Muslumanoglu' ${ }^{1}$, Abdullah İgci ${ }^{1}$, Yeşim Eralp ${ }^{5,6}$, Adnan Aydiner $^{5}$, Pınar Saip ${ }^{5}$, Ekrem Yavuz ${ }^{2}$ and Vahit Ozmen ${ }^{1}$

\begin{abstract}
Background: The expression of immune checkpoint receptors (ICRs) on tumor-infiltrating lymphocytes (TILs) is associated with better response to immunotherapies via immune checkpoint inhibitors. Therefore, we investigated various ICR expressions on TILs in patients with locally advanced triple-negative breast cancer (TNBC) after neoadjuvant chemotherapy (NAC).

Methods: Expressions of ICRs were examined immunohistochemically in surgical specimens ( $n=61)$ using monoclonal antibodies for PDL-1, PD-1, TIM-3, LAG-3, and CTLA-4. Positivity was defined as staining $\geq 1 \%$ on TILS.

Results: The median age was 49 (24-76) years. The majority of patients were clinically T3-4 $(n=31,50.8 \%)$ and clinically N1-3 ( $n=58,95.1 \%$ ) before NAC. Of those, $82 \%$ were found to have CTLA-4 positivity, whereas PD1, PDL-1, LAG3, and TIM-3 expressions on TILs were 62.3, 50.9, 26.2, and 68.9\%. A high expression of CTLA-4 was found to be associated with a better chemotherapy response ( $\mathrm{OR}=7.94,95 \% \mathrm{Cl}: 0.9-70.12, p=0.06)$, whereas TIM-3 positivity was contrarily associated with a worse chemotherapy response $(\mathrm{OR}=0.253,95 \% \mathrm{Cl}: 0.066-0.974, p=0.047)$ as measured by the MDACC Residual Cancer Burden Index. At a 47-month follow-up, ypNo (DFS; HR=0.31, 95\% Cl: $0.12-0.83, p=0.02$ and DSS; HR=0.21, 95\% Cl: $0.07-0.62, p=0.005$ ) and CTLA-4 high expression on TILS (DFS; HR= $0.38,95 \% \mathrm{Cl}: 0.17-0.85, p=0.019$ and DSS; HR $=0.34,95 \% \mathrm{Cl}: 0.15-0.78, p=0.01$ ) were found to be associated with improved survival.
\end{abstract}

Conclusions: These findings demonstrate that CTLA-4, PD-1, PDL-1, and TIM-3 were highly expressed in TNBC. Based on these high expression patterns, further studies directed towards combined therapies are warranted in advanced TNBC in future.

Keywords: Triple negative breast cancer, LAG-3, PD1, PDL1, CTLA4, T-cell immunoglobulin, Mucin domaincontaining molecule 3 (TIM3), Tumor infiltrating lymphocytes (TILs)

\footnotetext{
* Correspondence: neslicab@yahoo.com

'Department of General Surgery, Istanbul Medical Faculty, Breast Surgery

Service, Istanbul University, Istanbul, Turkey

Full list of author information is available at the end of the article
}

\section{$\triangle B M C$}

(c) The Author(s). 2021 Open Access This article is licensed under a Creative Commons Attribution 4.0 International License, which permits use, sharing, adaptation, distribution and reproduction in any medium or format, as long as you give appropriate credit to the original author(s) and the source, provide a link to the Creative Commons licence, and indicate if changes were made. The images or other third party material in this article are included in the article's Creative Commons licence, unless indicated otherwise in a credit line to the material. If material is not included in the article's Creative Commons licence and your intended use is not permitted by statutory regulation or exceeds the permitted use, you will need to obtain permission directly from the copyright holder. To view a copy of this licence, visit http://creativecommons.org/licenses/by/4.0/ The Creative Commons Public Domain Dedication waiver (http://creativecommons.org/publicdomain/zero/1.0/) applies to the data made available in this article, unless otherwise stated in a credit line to the data. 


\section{Background}

As the interaction between the immune system and tumor progression is better understood, new treatment strategies have been developed [1, 2]. A better understanding of this relationship has changed the treatment strategy for many cancers with the development of checkpoint inhibitors [3, 4]. Many recent studies have demonstrated that the expression of immune checkpoint receptors (ICRs) on tumor-infiltrating lymphocytes (TILs) is associated with better response to immunotherapies, including immune checkpoint inhibitors [4-8].

Cytotoxic $\mathrm{T}$ Lymphocyte antigen 4 (CTLA-4), Programmed Cell Death 1 (PD-1), and Programmed Cell Death Ligand 1 (PD-L1, also known as B7-H1 or CD274) pathways are the best known immune checkpoint pathways for immune responses within the tumor microenvironment. Although considerable research has been devoted to CTLA-4, PD-1, and PD-L1, less attention has been paid to $\mathrm{T}$ cell immunoglobulin and mucin domain-containing protein 3 (TIM-3) and Lymphocyteactivation gene 3 (LAG-3) [9]. The CTLA-4 antibody ipilimumab was the first checkpoint inhibitor for use in advanced melanoma [7]. Subsequently, the Food and Drug Administration (FDA) approved inhibitors of PD-1 and/or PD-L1 in different types of cancer. On the other hand, research on TIM-3- and LAG-3- based treatment options or combined checkpoint inhibitor therapy is still ongoing [10-14].

Triple-negative breast cancer (TNBC) constitutes about 15-20\% of all diagnosed breast cancers [15-17]. Although TNBC is typically characterized by a high degree of aggressiveness and poor prognosis, there are no approved molecularly targeted therapies for it $[18,19]$. A recent meta-analysis reported an improved outcome in early TNBC with pathologic complete response (pCR) receiving neoadjuvant chemotherapy (NAC) [20]. Sequential administration of anthracyclines followed by taxanes has been the most common neoadjuvant approach in TNBC, and addition of carboplatin has also been considered to increase the pCR especially in those with BRCA mutations [21]. On the other hand, recent literature stresses that TNBC is more immunogenic than other breast cancer types [22-24]. Expression of ICRs on TILs has been associated with a better response to immunotherapies including immune checkpoint inhibitors (ICIs) as shown in advanced metastatic breast cancer by using combined modalities with chemotherapy [25]. Based on these encouraging results, recent studies focused on intensive investigations in neoadjuvant setting in combinations of ICIs with chemotherapeutic agents including anthracyclines, taxanes and platinum [26].

While early-phase studies of the immune checkpoint inhibitors in TNBC have shown evidence of activity, it is still a matter of debate as to which immune checkpoint marker will be used in treatment or which immune checkpoint marker has a prognostic value. Therefore, we investigated the expression of various new emerging ICRs, including TIM-3 and LAG-3, along with the best known PDL-1, PD-1, and CTLA-4 in patients with TNBC receiving NAC to explore their predictive and prognostic significance.

\section{Methods}

The study included 61 patients with triple-negative locally advanced breast cancer (LABC) who underwent surgery following NAC at the Istanbul University, Faculty of Medicine, Department of General Surgery, from July 2002 to January 2018. The study was approved by the ethical committee of Istanbul University, Istanbul Medical Faculty.

\section{Patients and therapy}

Patients with residual disease and $\mathrm{pCR}$, who had received NAC due to the locally advanced TNBC were included in the study. The majority of patients $(n=57)$ received anthracycline and taxane containing NAC protocols, whereas $4(6.6 \%)$ had an anthracycline-based regimen and $5(6.6 \%)$ received additional carboplatine or cisplatine with/without gemcitabine or capecitabine. Of those, 45 (73.8\%) underwent mastectomy and 26 (26.2\%) had breast-conserving surgery. Furthermore, 53 patients (86.9\%) underwent axillary dissection with/without sentinel lymph node biopsy (SLNB), whereas only 8 patients (13.1\%) had SLN alone following completion of NAC. All patients received adjuvant radiotherapy to the chest wall for mastectomy and the whole breast for breastconserving surgery with axillary (level I-II-III) and supraclavicular lymph node region irradiation following surgery.

All patients with TNBC had estrogen receptor and progesterone receptor positivity $<1 \%$, and HER 2 negativity was determined according to the ASCO/CAP guidelines. The absence of a residual invasive tumor in the resected breast specimen and in the regional lymph nodes after completion of NAC was defined as pCR. The chemotherapy response was evaluated using the "MD Anderson Cancer Center (MDACC) Residual Cancer Burden Index" (www3.mdanderson.org/app/medcalc/ index.cfm.pagename $=$ jsconvert) [27]. The residual cancer burden index was calculated from the parameters in pathology reports, including primary tumor bed area size, overall cancer cellularity, and percentage of in situ cancer. The MDACC Residual Cancer Burden Index classification implies a worsening chemotherapy response from "Class 0" to "Class 3". "Class 0" was considered a pathological complete response, whereas "Class 3" was considered chemotherapy-resistant. Briefly, response to chemotherapy is considered good if Class 0 or 1 and 
not as good if Class 2 or 3. Clinicopathologic data included age, tumor characteristics, operation type, clinical and pathological stage, histologic grade, and lymph node status, and any local and/or systemic recurrences and follow-up time were analysed.

\section{Immunohistochemical analysis of immune checkpoint receptors}

Expression of ICRs was studied in paraffin blocks of surgical specimen following NAC, respectively. The most suitable tumor block that included tumor-infiltrating lymphocytes (TILs) was selected for immunostaining. Immunohistochemical evaluation was performed with an automatic Ventana BenchMark (Ventana Medical Systems, Tucson, AZ, USA) slide staining device. CTLA4, LAG-3, PD-L1, PD-1, and TIM-3 expression was assessed on $5-\mu \mathrm{m}$ formalin-fixed paraffin-embedded slides on tumor and TILs. Sections were incubated with primary antibodies for PDL-1 (rabbit mAb; SP263 clone kit, Ventana Medical Systems, Tucson, AZ, USA) at 1: 100 dilution, for PD-1 (rabbit mAb; AC0255RUO, Epitomics, Abcam, UK) at 1:200 dilution, for CTLA-4 (rabbit policlonal Ab; PA5-23967, Thermo Fisher Scientific, UK) at 1:100 dilution, for LAG-3 (rabbit policlonal Ab D2G40" Cell Signaling Tech., MA, USA) at 1:200 dilution, and for TIM-3 (XP rabbit, D5D5R ${ }^{\mathrm{Tm}}$, Cell Signaling Tech., MA, USA) at 1:200 dilution. Tonsil tissue was used as a control in the immunohistochemistry procedure for all of these antibodies.

The intensity and staining percentage were noted for each immune checkpoint receptor showing membranous staining. The intensity was scored as weak, moderate, or strong. Different staining percentages from 1 to $20 \%$ depending on the median values for each marker with or without staining intensity were tested for any significant associations with prognosis. CTLA-4 and PD-L1 positivity were defined as membranous staining $>1 \%$ on either tumor or TILs, whereas LAG-3, TIM-3, and PD-1 positivity on TILs was defined as membranous staining $\geq 1 \%$ on TILs. All micrographs were taken by using an integrated digital camera (Olympus DP71, Japan) on a light microscope (Olympus BX51, Japan).

\section{Statistical analysis}

The statistical analyses were performed using the statistical software program SPSS 25 (Statistical Package for Social Sciences; SPSS, IBM Corp., Armonk, NY, USA). A $p$-value equal to or less than 0.05 was considered statistically significant. Categorical variables were evaluated by Chi Fisher's exact tests including Pearson chi-square, continuity correction, and Fisher's exact tests in twotailed univariate analyses. Binary logistic regression analysis was used to estimate the significant associations linked to chemotherapy response. Furthermore, the
Pearson correlation test was used to assess the correlations of continuous variables.

Disease-free and disease-specific survival rates were calculated by Kaplan-Meier analyses, and factors associated with survival were tested by the log-rank test. Disease-free survival (DFS) was analysed by considering local and systemic metastases, and disease-specific survival (DSS) rates were analysed by considering breastcancer-related mortality. A log-rank test was used to compare the prognostic effect of different variables. Variables that were found to be significant in univariate analyses were further evaluated in a Cox regression model to calculate the hazard ratio of factors associated with poor prognosis.

\section{Results}

The patients' median age was 49 (24-76) years. Of 61 patients, $31(50.8 \%)$ were clinically T3-4 (55\%), whereas the remainder were cT1-2 (49.2\%). Almost all of them were clinically N1-3 $(n=58,95.1 \%)$ presenting mostly with $\mathrm{cN} 1$ disease $(n=36,59 \%)$. Twenty-three patients (37.7\%) were found to have axillary pathologic complete response (ypN0), while 18 had (29.5\%) ypN1, 11 (18\%) had ypN2, and 9 (14.8\%) had ypN3 after NAC. Among patients having a residual tumor $(n=51)$, the vast majority had invasive ductal carcinoma $(n=41,80.4 \%)$, while 3 (5.9\%) had invasive lobular carcinoma, 1 (1.9\%) had a mix of invasive ductal and lobular, and 6 (11.8\%) had metaplastic carcinoma.

Response to chemotherapy and histopathological analysis was evaluated in 60 patients by the "MDACC Residual Cancer Burden Index". One patient with a residual tumor in the lymphovascular area was excluded from this analysis. The median score was $2.72(0-5.1)$. According to the MDACC Residual Cancer Burden classification, 26 patients (43.3\%) had Class III as nonresponders, 21 patients (35\%) had Class II as a partial response, $4(6.7 \%)$ had Class I as a near-complete response, and 9 (15\%) had Class 0 with a pathologic complete response.

\section{Immunohistochemical expression of immune checkpoint receptors}

The median values of staining percentages for PD-L1, PD-1, CTLA-4, LAG-3, and TIM-3 in TILs were $1(0$ $50), 2(0-70), 7.5(0-60), 0(0-60)$, and $2(0-50)$, respectively, whereas the staining percentages of tumoral expressions of PD-L1 and CTLA-4 were $0.5(0-30)$ and $7.5(0-70)$, respectively.

Significant correlations were detected between different immune checkpoint receptors on TILs in the Spearman correlation analysis (Table 1). Expression levels of PD-L1 on TILs were significantly correlated with PD-L1 expression on the tumor $(\mathrm{p}=<0.001)$, and PD-1 $(p=$ 
Table 1 Spearman correlations (SC) of the immune checkpoint receptor (=ICR) expressions in TILs $(n=61)$

\begin{tabular}{|c|c|c|c|c|c|c|c|c|}
\hline & & PDL-1 & PDL-1 $1_{\text {tumor }}$ & PD1 & LAG3 & TIM3 & CTLA-4 TILs & CTLA-4 ${ }_{T M}$ \\
\hline \multirow[t]{3}{*}{ PDL-1 tumor $_{\text {tu }}$} & $r$ & 0.772 & & & & & & \\
\hline & P-value & $<0.001^{* *}$ & & & & & & \\
\hline & $n$ & 48 & & & & & & \\
\hline \multirow[t]{3}{*}{ PD1 } & $r$ & 0.354 & 0.352 & & & & & \\
\hline & $P$-value & $0.007^{* *}$ & $0.014^{*}$ & & & & & \\
\hline & $\mathbf{n}$ & 57 & 48 & & & & & \\
\hline \multirow[t]{3}{*}{ LAG3 } & $r$ & 0.370 & 0.476 & 0.174 & & & & \\
\hline & $P$-value & $0.005^{* *}$ & $0.001^{* *}$ & 0.179 & & & & \\
\hline & $n$ & 57 & 48 & 61 & & & & \\
\hline \multirow[t]{3}{*}{ TIM3 } & $r$ & 0.256 & 0.236 & 0.407 & 0.274 & & & \\
\hline & P-value & 0.055 & 0.106 & $0.001^{* *}$ & $0.033^{*}$ & & & \\
\hline & $n$ & 57 & 48 & 61 & 61 & & & \\
\hline \multirow[t]{3}{*}{ CTLA-4 $4_{\text {ILs }}$} & $r$ & 0.148 & 0.068 & 0.460 & 0.037 & 0.301 & & \\
\hline & $P$-value & 0.272 & 0.648 & $<0.001^{* *}$ & 0.776 & $0.018^{*}$ & & \\
\hline & $\mathrm{n}$ & 57 & 48 & 61 & 61 & 61 & & \\
\hline \multirow[t]{3}{*}{ CTLA-4 TM } & $r$ & 0.131 & 0.106 & 0.248 & 0.251 & 0.277 & 0.486 & \\
\hline & $P$-value & 0.374 & 0.472 & 0.076 & 0.073 & $0.047^{*}$ & $<0.001 * *$ & \\
\hline & $n$ & 48 & 48 & 52 & 52 & 52 & 52 & \\
\hline \multirow[t]{3}{*}{ MDACC Score } & $r$ & -0.061 & -0.010 & 0.153 & -0.061 & 0.062 & 0.008 & 0.119 \\
\hline & $P$-value & 0.653 & 0.947 & 0.244 & 0.645 & 0.637 & 0.953 & 0.405 \\
\hline & $\mathbf{n}$ & 56 & 47 & 60 & 60 & 60 & 60 & 51 \\
\hline
\end{tabular}

${ }^{*} p<0.05,{ }^{* *} p<0.01$, r: Spearman Correlations

MDARB-Index: MD Anderson Residual Cancer Burden Index

$0.007)$, LAG-3 $(p=0.005)$, whereas PD-L1 expression on TILs was found to be correlated with TIM-3 expression levels on TILs $(p=0.055)$ that did not reach the statistical significance (Table 1). Expression of PD-1 has also shown significant correlations with CTLA-4 $(p<0.001)$ and TIM- 3 on TILs ( $p=0.001)$, whereas CTLA- 4 expression on TILs correlated with CTLA-4 expression on the tumor $(\mathrm{p}<0.001)$ and TIM-3 expression on TILs ( $p=$ 0.018). Finally, LAG-3 expression on TILs was found to be significantly correlated with TIM-3 expression on TILs $(p=0.033)$.

A cut-off $\geq 1 \%$ staining percentage regardless of staining intensity was considered positive for each ICR. Of 61 cases, TIL-associated positivities for the ICRs were as follows: PD-L1 (50.9\%), PD-1 (62.3\%), LAG-3 (26.2\%), TIM-3 (52\%), and CTLA-4 (82\%) (Table 2, Fig. 1). The higher levels of staining percentages based on the median values of each marker with/without staining intensity were tested for any significant associations with survival. High CTLA-4 expression was considered for any staining $\geq 15 \%$, and any medium and strong staining if $<15 \%$.

Associations of ICR positivities and response to chemotherapy were tested by using the MDACC
Residual Cancer Burden Index. A high expression of CTLA-4 $(p=0.047)$ and TIM-3-negativity $(p=0.003)$ were found to be significantly associated with $\mathrm{pCR}$ (Table 2). When the MDACC Residual Cancer Burden Index "Class 0\&1" vs "Class $2 \& 3$ " were compared, high expression of CTLA-4 on TILs was found to be associated with a better chemotherapy response $(\mathrm{OR}=7.94$, 95\% CI: $0.9-70.12, p=0.06$ ), whereas TIM-3 positivity was contrarily associated with a worse chemotherapy response $(\mathrm{OR}=0.253,95 \% \mathrm{CI}$ : $0.066-0.974, p=0.047)$. No significant associations could be found between other ICR expressions and response to NAC.

\section{Outcome and survival analyses}

At the median follow-up time of 47 months (12-204), the 5-year disease-free survival (DFS) and diseasespecific survival (DSS) were 55 and $59.8 \%$ for the whole cohort, respectively. Associations between clinicodemographic and pathological characteristics and survival revealed that patients with ypNO or a pCR or with a Class 0\&I MDACC Residual Cancer Burden Index indicating a good response to NAC were found to have a better 5year disease-free survival (DFS) and disease-specific survival (DSS) (Table 3, Fig. 2). Positivities of PD-L1, PD-1, 
Table 2 Associations of immune check point receptor expression in TILs with neoadjuvant chemotherapy response as calculated "MD Anderson Cancer Center (MDACC) Residual Cancer Burden Index" in TNBC patients

\begin{tabular}{|c|c|c|c|c|c|c|c|}
\hline $\begin{array}{l}\text { Expression in } \\
\text { TILs }(\geq \% 1)\end{array}$ & $\mathrm{N}=61(\%)$ & pCR(+) vs $(-)^{a}$ & $P$-value & Class $0 / \mathrm{l}$ vs $\mathrm{II} / \mathrm{II}^{\mathrm{a}}$ & $P$-value & Class $0 / \mathrm{I} / \mathrm{II}$ vs III & $P$-value \\
\hline PD-L1 (+) vs other & $29 / 57(50.9 \%)$ & $5 / 9(56 \%)$ vs $24 / 47$ (51\%) & 0.999 & $6 / 11(55 \%)$ vs $23 / 45(51 \%)$ & 0.999 & $19 / 31$ (61\%) vs 10/25 (40\%) & 0.188 \\
\hline PD1 (+) vs other & $37 / 61(62 \%)$ & $5 / 9(56 \%)$ vs $32 / 51$ (63\%) & 0.721 & $7 / 13(54 \%)$ vs 30/47 (64\%) & 0.739 & $19 / 34(56 \%)$ vs $18 / 26(69 \%)$ & 0.432 \\
\hline LAG3 (+) vs other & $16 / 61(26 \%)$ & $2 / 9$ (22\%) vs $14 / 51$ (28\%) & 0.999 & $3 / 13(23 \%)$ vs $13 / 47(28 \%)$ & 0.999 & $11 / 34(32 \%)$ vs $5 / 26(19 \%)$ & 0.378 \\
\hline PD1\&LAG3 (+) vs other & $14 / 61(23 \%)$ & $2 / 9$ (22\%) vs $12 / 51$ (24\%) & 0.999 & $3 / 13(23 \%)$ vs $11 / 47(23 \%)$ & 0.999 & $9 / 34(26 \%)$ vs $5 / 26(19 \%)$ & 0.555 \\
\hline TIM3 (+) vs other & $42 / 61(69 \%)$ & $2 / 9$ (22\%) vs $39 / 51$ (77\%) & 0.003 & $6 / 13(46 \%)$ vs $35 / 47$ (75\%) & 0.108 & $25 / 34(74 \%)$ vs $16 / 26(62 \%)$ & 0.478 \\
\hline PD1\&TIM3 (+) vs other & $31 / 61(51 \%)$ & $2 / 9$ (22\%) vs $28 / 51$ (55\%) & 0.145 & $4 / 13$ (31\%) vs $26 / 47$ (55\%) & 0.209 & $16 / 34(47 \%)$ vs $14 / 26(54 \%)$ & 0.794 \\
\hline CTLA-4 (+) vs other & $50 / 61(82 \%)$ & 9/9 (100\%) vs 40/51 (78\%) & 0.189 & $12 / 13(92 \%)$ vs $37 / 47(79 \%)$ & 0.427 & $30 / 34(88 \%)$ vs $19 / 26(73 \%)$ & 0.182 \\
\hline CTLA- $4^{\mathrm{b}}(+)$ vs other & $43 / 61(70.5 \%)$ & $9 / 9(100 \%)$ vs $33 / 51$ (65\%) & 0.047 & $12 / 13(92 \%)$ vs $30 / 47(64 \%)$ & 0.084 & $26 / 34(77 \%)$ vs $16 / 26(62 \%)$ & 0.334 \\
\hline PD1\&CTLA-4 (+) vs other & $33 / 61(54 \%)$ & $5 / 9(56 \%)$ vs $27 / 51(53 \%)$ & 0.999 & $7 / 13(54 \%)$ vs $25 / 47(53 \%)$ & 0.999 & $18 / 34(53 \%)$ vs $14 / 26(54 \%)$ & 0.999 \\
\hline
\end{tabular}

Tumor infiltrating (stromal) lymphocytes (=TILs)

a One patient with a residual tumor in the lymphovascular area has been excluded

${ }^{b}$ CTLA-high expression: $\geq 15 \% \&$ any moderate or strong staining if $<15 \%$

LAG-3, TIM-3, and CTLA4 and different combinations of ICR coexpressions including PD-L1/LAG3, etc. and different cut-off levels based on staining percentage and/ or staining intensity were tested for any significant associations with survival as shown in Table 4. High CTLA4 expression on TILs was the only significant factor associated with an improved 5-year disease-free survival (DFS) and disease-specific survival (DSS) (DFS: CTLA4- low, 36.5\% vs CTLA4-high, 63.7\%; $p=0.043$; DSS: CTLA4-low, 43.2\% vs CTLA4-high, $73.5 \% ; p=0.017$ ) (Fig. 3). A multivariate logistic regression analysis demonstrated that ypNO (DFS; HR $=0.31,95 \%$ CI: $0.12-0.83$, $p=0.02$ and DSS; $\mathrm{HR}=0.21,95 \%$ CI: $0.07-0.62, p=$ 0.005 ) and CTLA-4 high expression (DFS; HR $=0.38$, 95\% CI: $0.17-0.85, p=0.019$ and DSS; HR $=0.34,95 \%$ CI: $0.15-0.78, p=0.01$ ) were found to be associated with

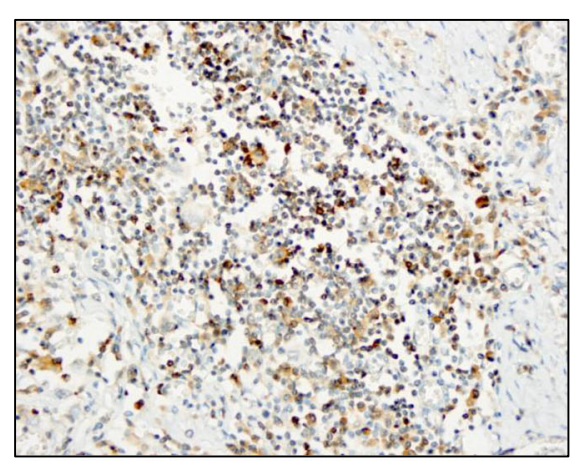

a

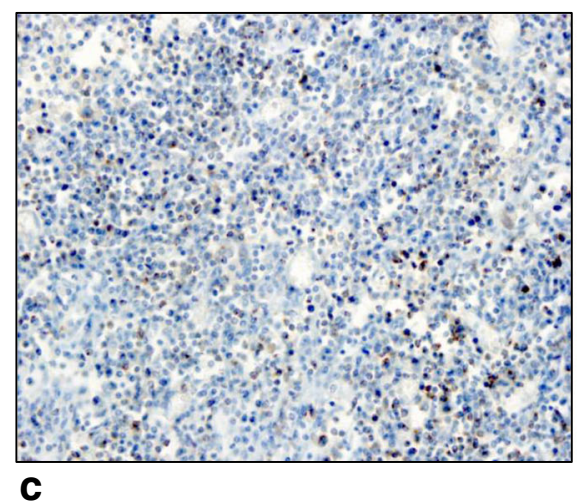

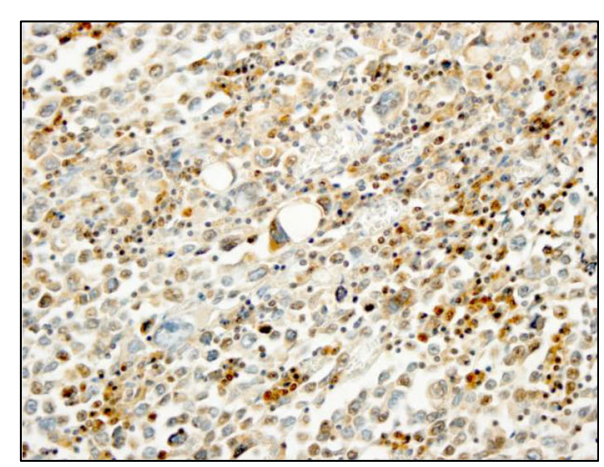

b

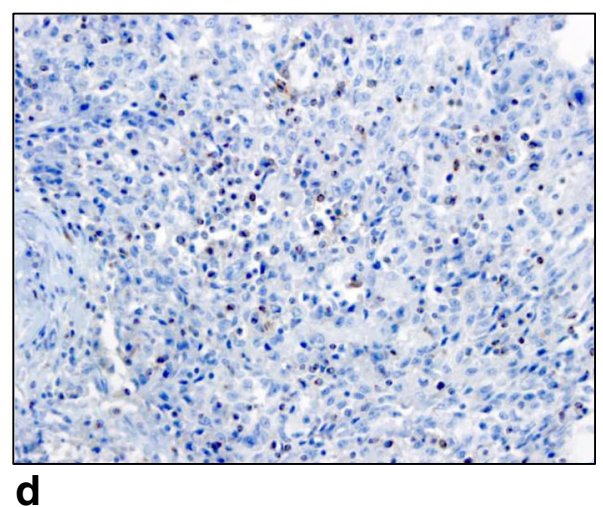

Fig. 1 a. High PD1 immunohistochemical expression on TILs ( $\times$ 400, HPF. b. High CTLA4 immunohistochemical expression on TILs and tumor ( $\times$ 400, HPF). c. Immunohistochemical expression of LAG-3 on TILs ( $\times$ 400, HPF). d. Immunohistochemical expression of TIM3 on TILs ( $\times$ 400, HPF) 
Table 3 Survival analyses associated with clinicodemographic or pathological patient characteristics

\begin{tabular}{|c|c|c|c|c|c|}
\hline Characteristics & $\mathrm{n}$ & 5-Year DFS(\%) & $\mathbf{p}$ & 5 Year DSS(\%) & $\mathbf{p}$ \\
\hline Age $\leq 50$ & 31 & 55.8 & 0.999 & 58.2 & 0.895 \\
\hline Age $>50$ & 30 & 54.6 & & 61.4 & \\
\hline cT (Clinical = Physical exam\&Radiological) & & & 0.624 & & 0.526 \\
\hline $\mathrm{T} 1-2$ & 30 & 55.7 & & 62.9 & \\
\hline T3-4 & 31 & 53.9 & & 57.2 & \\
\hline $\mathrm{cN}$ & & & 0.719 & & 0.441 \\
\hline No-1 & 39 & 55.6 & & 64.0 & \\
\hline N2-3 & 22 & 52.3 & & 52.1 & \\
\hline ypN* & & & $0.031 *$ & & $0.008^{*}$ \\
\hline ypNO & 23 & 73.9 & & 78.0 & \\
\hline ypN1-3 & 38 & 43.6 & & 48.1 & \\
\hline Pathological findings after NAC & & & 0.367 & & 0.230 \\
\hline Invasive ductal carcinoma & 40 & 48.0 & & 54.6 & \\
\hline Other & 21 & 66.0 & & 69.7 & \\
\hline *MDACC Residual Cancer Burden Index & & & $0.049 *$ & & $0.041 *$ \\
\hline Class 0 (=pathologic complete response) & 9 & 88.9 & & 88.9 & \\
\hline Class I-II-III & 51 & 49.3 & & 53.0 & \\
\hline *MDACC Residual Cancer Burden Index & & & $0.038^{*}$ & & $0.037^{*}$ \\
\hline Class 0-1 (=pathologic complete response) & 13 & 83.9 & & 83.9 & \\
\hline Class II-III & 47 & 48.1 & & 52.7 & \\
\hline
\end{tabular}

NAC Neoadjuvant chemotherapy, ${ }^{*} p<0.05$

a good prognosis (Table 5, Fig. 3). No significant difference could be found in DFS and DSS rates in regards to PD-L1, PD-1, TIM-3, and LAG-3 expressions on TILs (Table 4, Fig. 4). Furthermore, CTLA4 tumoral expression $(\geq 1 \%)$ was seen in 25 of 52 patients with residual tumor after NAC (48\%). No difference could be found between patients with CTLA4-positivity and CTLA4negativity on tumor in 5-year DFS (CTLA-4(+), 51.4\% vs CTLA-4(-), 53\%, $p=0.846)$ and DSS rates (CTLA4(+), $51.1 \%$ vs CTLA4(-), $58.2 \%, p=0.814)$.

\section{Discussion}

Targeting immune checkpoints in cancer immunotherapy has made substantial progress in the treatment of certain solid cancers, including breast cancer, focusing on TNBC as the most immunogenic and aggressive subtype. However, long-term durable responses were obtained in only 10 to $30 \%$ of patients, while some patients develop resistance to the current immunotherapy regimens and may benefit from further combinatorial blockade targeting potential new immune checkpoints, such as LAG-3 or TIM-3 [28]. Furthermore, a significant proportion of patients suffer from immune-related adverse effects when treated with CTLA-4, PD-1, or PD-L1 inhibitors. The hypothesis regarding whether combining the currently known inhibitors targeting PD-1, PD-L1, or CTLA-4 with the promising new inhibitors against LAG-3 and TIM-3 may enhance therapeutic efficacy while reducing the side effects is currently under investigation in clinical trials [14]. In this study, the predictive and prognostic significance of various ICRs including PDL1, PD-1, and CTLA-4, along with the new emerging receptors TIM3 and LAG3, was tested in locally advanced TNBC. We briefly report here that CTLA-4 was the only biomarker as ICR associated with a better chemotherapy response and better outcome, whereas TIM-3 expression was negatively correlated with response to NAC.

Multiple ICRs, including PD-1, CTLA-4, TIM-3, and LAG-3, were found to be upregulated in breast cancer tissues as compared to normal breast tissue [29]. Despite some inconsistent results in the literature, the majority of the studies indicated an improved prognosis and better response to immunotherapy in patients with TNBC having PD-1 or PD-L1 expression on TILs $[30,31]$. In a recent meta-analysis including 47 studies with a total of 14 , 367 patients who had breast cancer, Huang et al. [31] reported that PD-L1+ expression on TILs was 


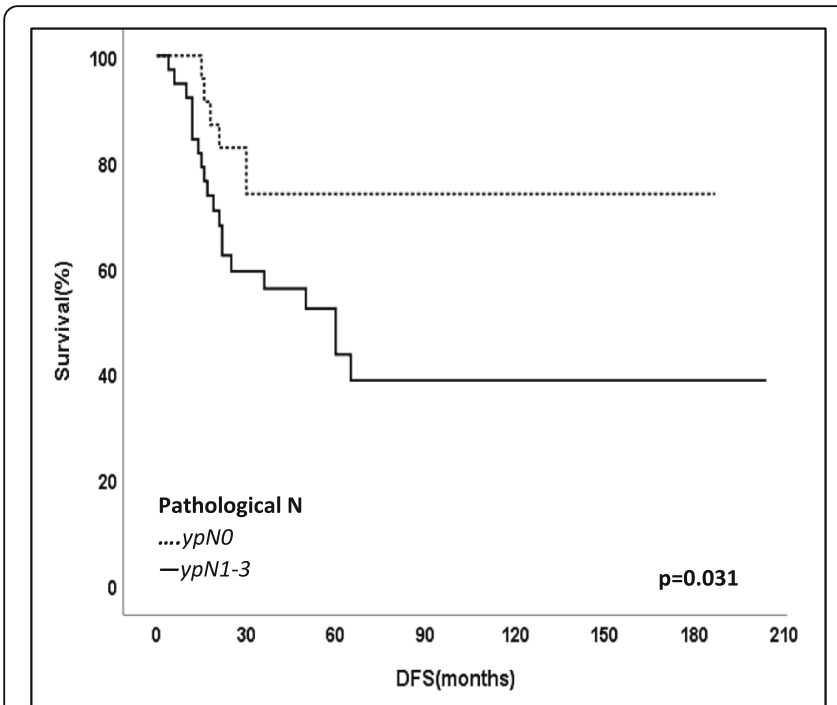

a

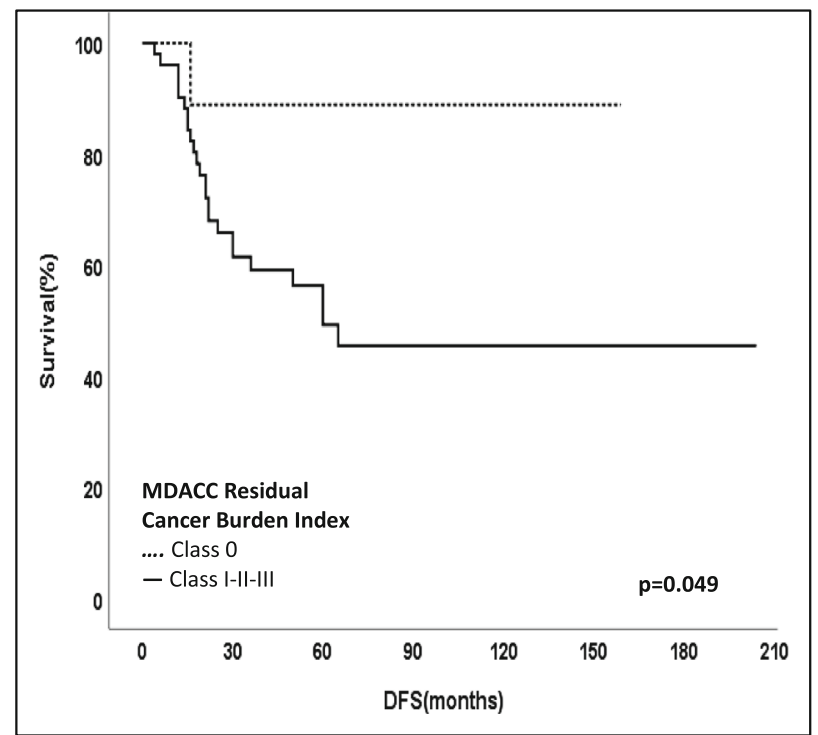

C

Fig. 2 a. 5-year disease-free survival (DFS) in patients with ypN0, 73.9\% vs ypN+, 43.6\%; $p=0.031$. b. 5 -year disease-specific survival (DSS) in patients with ypN0, 78\% vs ypN+, 48.1\%; $p=0.008$. c. 5-year disease-free survival (DFS) in patients with MDACC Residual Cancer Burden Index Class 0, 88.9\% vs MDACC Residual Cancer Burden Index Class I-III, 49.3\%; $p=0.049$. d. 5 -year disease-free survival (DSS) in patients with MDACC Residual Cancer Burden Index Class 0, 88.9\% vs MDACC Residual Cancer Burden Index Class I-III, 53\%; $p=0.041$

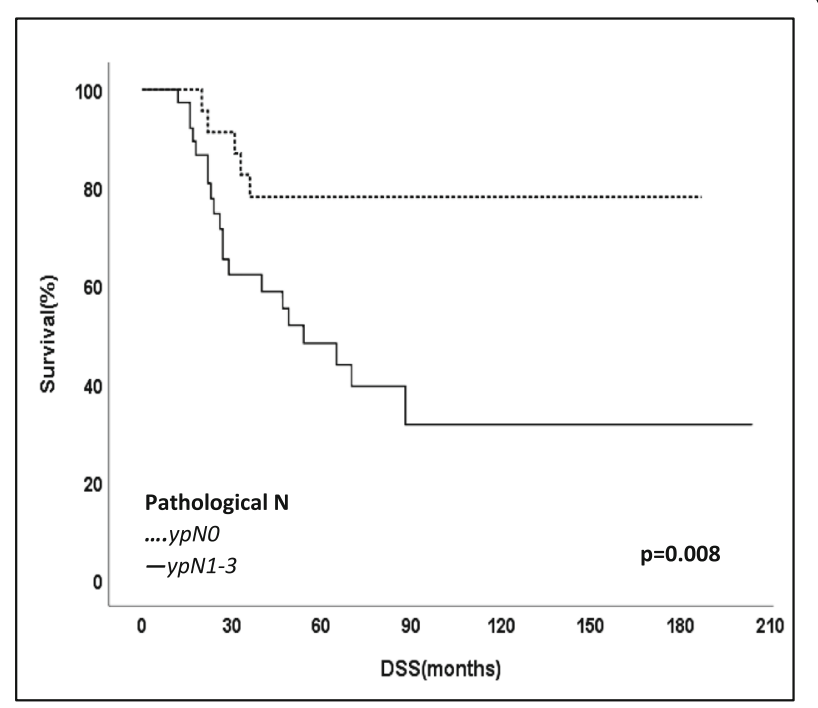

b

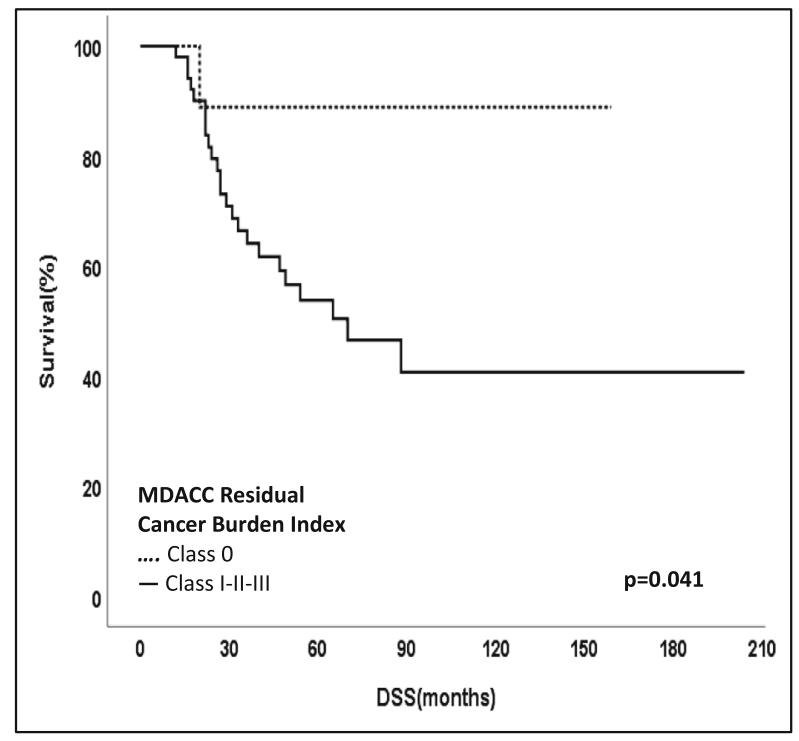

d associated with a better DFS (HR $=0.45,95 \% \mathrm{CI}$ : $0.28-0.73, p=0.001)$ and $\mathrm{OS}(\mathrm{HR}=0.41,95 \% \mathrm{CI}$ : $0.27-0.63, p<0.0001)$. Contrarily, PD-L1 expression on the tumor was found to be associated with shorter DFS $(\mathrm{HR}=1.43,95 \%$ CI: $1.21-1.70, \mathrm{p}<$ 0.0001 ) and overall survival (OS, $\mathrm{HR}=1.58,95 \% \mathrm{CI}$ : $1.14-2.20, p=0.006$ ). In our study, we could not find any significant difference in DFS or DSS between patients with or without PD-L1 or PD-1 expression on TILs. Though there was a trend towards a better prognosis in patients with PD-L1 expression on TILs, it did not reach statistical significance.
Furthermore, we found a significant correlation between tumoral PD-L1 expression and PD-1 expression on TILs similar to the findings of the metaanalysis of Huang et al. [31].

The first inhibitory immune checkpoint receptor studied in the clinic, CTLA-4, was previously shown to be present as a surface molecule on solid tumor cell lines including breast carcinomas and osteosarcomas [32]. Though the exact function of expression of CTLA-4 on tumor cells is unknown, incubating the cells with CTLA-4 ligands CD80 or CD86 induced apoptosis in human osteosarcoma cell line 
Table 4 Survival analyses associated with immune check point receptor expression in patients with TNBC after NAC

\begin{tabular}{|c|c|c|c|c|c|}
\hline Expressions in TILs & $\mathrm{N}$ & $\begin{array}{l}\text { 5-year Disease Free } \\
\text { Survival (DFS) (\%) }\end{array}$ & $\mathrm{p}$ & $\begin{array}{l}\text { 5-year Disease Specific } \\
\text { Survival (DSS) (\%) }\end{array}$ & $p$ \\
\hline PDL-1 $\geq 1 \%(+)$ Vs < $1 \%(-)$ & $29 / 28$ & $62.5 \%$ vs $39.5 \%$ & 0.141 & $67.2 \%$ vs $50.4 \%$ & 0.132 \\
\hline PDL-1 $\geq 5 \%(+)$ Vs < $5 \%(-)$ & $25 / 32$ & $65.3 \%$ vs $38.9 \%$ & 0.096 & $66.2 \%$ vs $53.3 \%$ & 0.213 \\
\hline PDL-1 High $(\geq 10 \%)$ vs Low $(<10 \%)^{a}$ & $26 / 31$ & $66.2 \%$ vs $37.5 \%$ & 0.062 & $67.6 \%$ vs $51.5 \%$ & 0.145 \\
\hline PDL-1 $\geq 10 \%(+)$ Vs $<10 \%(-)$ & $18 / 39$ & $67.4 \%$ vs $42.9 \%$ & 0.087 & $69.9 \%$ vs $54.0 \%$ & 0.197 \\
\hline PDL-1 $\geq 20 \%(+)$ vs < 20\% (-) & $15 / 42$ & $50.0 \%$ vs $51.3 \%$ & 0.996 & $51.9 \%$ vs $62.1 \%$ & 0.651 \\
\hline PD-1 $\geq 1 \%(+)$ Vs < $1 \%(-)$ & $38 / 23$ & $50.6 \%$ vs $61.6 \%$ & 0.121 & $51.4 \%$ vs $62.7 \%$ & 0.082 \\
\hline PD-1 $\geq 5 \%(+)$ vs < $5 \%(-)$ & $26 / 35$ & $54.2 \%$ vs $56.1 \%$ & 0.593 & $58.1 \%$ vs $69.1 \%$ & 0.270 \\
\hline PD-1 $\operatorname{High}(\geq 10 \%)$ vs $\operatorname{Low}(<10 \%)^{a}$ & $24 / 37$ & $56.8 \%$ vs $54.8 \%$ & 0.517 & $59.6 \%$ vs $66.9 \%$ & 0.263 \\
\hline PD-1 $\geq 10 \%(+)$ vs < 10\% (-) & $10 / 51$ & $67.5 \%$ vs $49.4 \%$ & 0.193 & $72.9 \%$ vs $57.4 \%$ & 0.439 \\
\hline PD-1 $\geq 20 \%(+)$ vs < 20\% (-) & $6 / 55$ & $83.3 \%$ vs $52.2 \%$ & 0.277 & $83.3 \%$ vs $59.5 \%$ & 0.570 \\
\hline LAG-3 $\geq 1 \%(+)$ vs < $1 \%(-)$ & $16 / 45$ & $68.8 \%$ vs $50.8 \%$ & 0.370 & $60.6 \%$ vs $59.4 \%$ & 0.778 \\
\hline LAG-3 $\geq 5 \%(+)$ vs $<5 \%(-)$ & $7 / 54$ & $57.1 \%$ vs $55.3 \%$ & 0.804 & $71.4 \%$ vs $62.9 \%$ & 0.339 \\
\hline LAG-3 $\operatorname{High}(\geq 10 \%)$ vs Low $(<10 \%)^{a}$ & $6 / 55$ & $66.7 \%$ vs $53.9 \%$ & 0.815 & $66.7 \%$ vs $61.3 \%$ & 0.683 \\
\hline TIM3 $\geq 1 \%(+)$ vs < $1 \%(-)$ & $42 / 19$ & $50.5 \%$ vs $65.6 \%$ & 0.473 & $53.6 \%$ vs $72.3 \%$ & 0.524 \\
\hline TIM3 $\geq 5 \%(+)$ vs < 5\% (-) & $15 / 46$ & $52.8 \%$ vs $55.4 \%$ & 0.947 & $62.7 \%$ vs $59.2 \%$ & 0.959 \\
\hline TIM3 High $(\geq 10 \%)$ vs Low $(<10 \%)^{a}$ & $14 / 47$ & $57.1 \%$ vs $55.2 \%$ & 0.314 & $68.8 \%$ vs $60.5 \%$ & 0.535 \\
\hline CTLA-4 $\geq 1 \%(+)$ vs $<1 \%(-)$ & $50 / 11$ & $57.2 \%$ vs $45.5 \%$ & 0.272 & $63.2 \%$ vs $45.5 \%$ & 0.209 \\
\hline CTLA-4 $\geq 5 \%(+)$ vs < 5\% (-) & $48 / 13$ & $55.9 \%$ vs $53.8 \%$ & 0.595 & $61.8 \%$ vs $53.8 \%$ & 0.481 \\
\hline CTLA-4 High( $\geq 15 \%)$ vs Low $(<15 \%)^{b}$ & $43 / 18$ & $63.7 \%$ vs $36.5 \%$ & 0.043 & $70.5 \%$ vs $43.2 \%$ & 0.017 \\
\hline CTLA-4 $\geq 10 \%(+)$ vs $<10 \%(-)$ & $29 / 32$ & $60.1 \%$ vs $51.1 \%$ & 0.429 & $67.4 \%$ vs $53.6 \%$ & 0.468 \\
\hline CTLA-4 $\geq 15 \%(+)$ vs $<15 \%(-)$ & $19 / 42$ & $74.9 \%$ vs $46.6 \%$ & 0.103 & $74.0 \%$ vs $53.8 \%$ & 0.245 \\
\hline CTLA-4 $\geq 20 \%(+)$ vs $<20 \%(-)$ & $18 / 43$ & $72.9 \%$ vs $48.1 \%$ & 0.165 & $72.1 \%$ vs $55.0 \%$ & 0.373 \\
\hline PD1/PDL-1 $\geq 1 \%(+)$ vs $<1 \%(-)$ & $22 / 38$ & $52.5 \%$ vs $51.3 \%$ & 0.908 & $58.4 \%$ vs $60.4 \%$ & 0.997 \\
\hline PD1/LAG3 $\geq 1 \%(+)$ vs $<1 \%(-)$ & $14 / 47$ & $64.3 \%$ vs $52.4 \%$ & 0.619 & $64.0 \%$ vs $61.1 \%$ & 0.903 \\
\hline PD1/TIM3 $\geq 1 \%(+)$ Vs $<1 \%(-)$ & $31 / 30$ & $48.5 \%$ vs $62.2 \%$ & 0.273 & $49.4 \%$ vs $75.2 \%$ & 0.204 \\
\hline PD1/CTLA4 $\geq 1 \%(+)$ vs $<1 \%(-)$ & $33 / 28$ & $58.6 \%$ vs $50.2 \%$ & 0.966 & $59.9 \%$ vs $65.0 \%$ & 0.984 \\
\hline
\end{tabular}

${ }^{a}>\% 10 \& a n y$ moderate/strong staining vs other

b $\geq \% 15$ \&any moderate/strong staining vs other bold: $p \leq 0.05$

HOS cells through caspase- 3 and caspase- 8 activation [32]. Yu et al. showed that CTLA-4+ lymphocyte density high was an independent predictor of better DFS (HR $=0.315,95 \% \mathrm{CI}: 0.15-0.658, p=0.002)$ and OS $(\mathrm{HR}=0.313,95 \%$ CI: $0.139-0.703, \quad p=0.005)$, whereas tumor CTLA-4 ${ }^{\text {high }}$ was an independent predictor of shorter DFS $(\mathrm{HR}=2.176,95 \% \mathrm{CI}$ : 1.084-4.437, $p=0.029)$ and $\mathrm{OS}(\mathrm{HR}=2.820,95 \% \mathrm{CI}$ : $1.337-5.95, p=0.007)$ in 130 patients with operable breast cancer [33]. In our study, almost half of the tested tumors expressed tumoral CTLA-4 that significantly correlated with CTLA-4 and TIM3 expression on TILs. In concordance with the study of $\mathrm{Yu}$ et al., we demonstrated that CTLA-4 high expression on TILs (DFS; HR $=0.38,95 \%$ CI: $0.17-0.85, p=$
0.019 and DSS; $\mathrm{HR}=0.34,95 \%$ CI: 0.15-0.78, $\mathrm{p}=$ 0.01 ) and ypN0 (DFS; HR $=0.31,95 \%$ CI: $0.12-0.83$, $\mathrm{p}=0.02$ and DSS; HR $=0.21,95 \% \mathrm{CI}: 0.07-0.62, \mathrm{p}=$ 0.005) were independently found to be associated with improved survival in multivariate analysis. However, we could not find any prognostic significance of tumoral CTLA-4 expression in our study consisting of patients with locally advanced TNBC, whereas Yu's study included operable patients with both luminal and non-luminal tumors. Furthermore, high expression of CTLA-4 on TILs was significantly associated with a $\mathrm{pCR}$ and a better chemotherapy response (OR $=7.94,95 \% \mathrm{CI}: 0.9-70.12, p=0.06)$ based on the MDACC Residual Cancer Burden Index in our cohort with TNBC. Similarly, Kaewkangsadan 


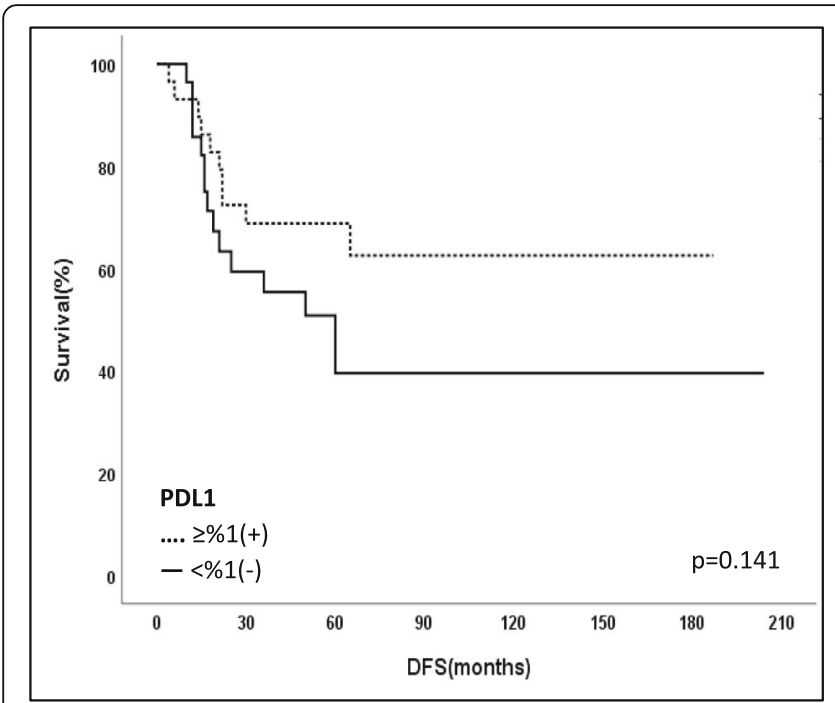

a

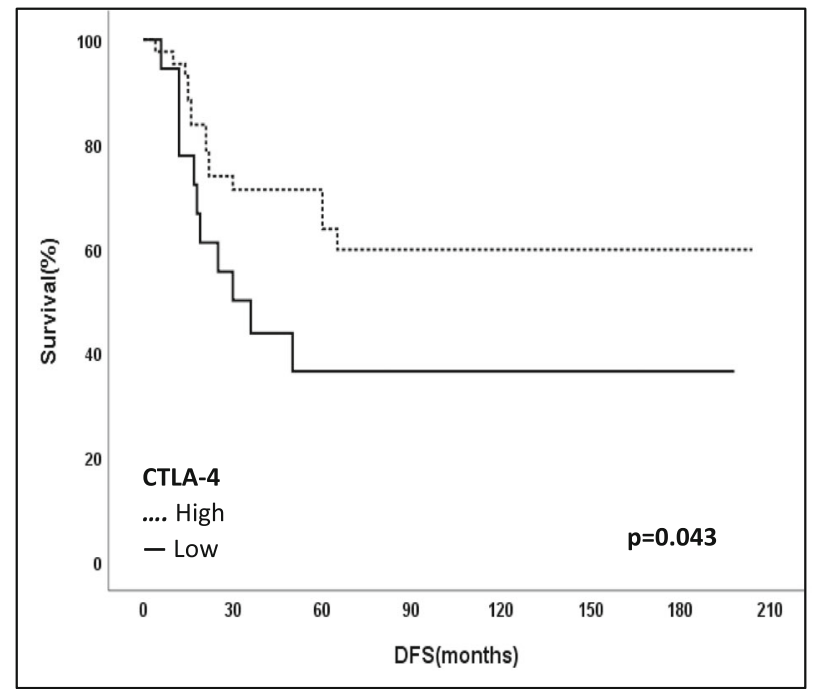

C

Fig. 3 a. 5 -year disease-free survival (DFS) in patients with PDL-1 $(\geq 1 \%), 62.5 \%$, vs PDL-1 $(<1 \%), 39.5 \%$; $p=0.141$. b. 5 -year disease-specific survival (DSS) in patients with PDL-1 $(\geq 1 \%), 67.2 \%$, vs PDL-1 $(<1 \%), 50.4 \% ; p=0.132$. c. 5 -year disease-free survival (DFS) in patients with CTLA4-High*, $63.7 \%$, vs CTLA4-Low, 36.5\%; $p=0.043{ }^{*} \geq 15 \%$ \& any moderate/strong staining if $\left.<15 \%\right)$. d. 5 -year disease-specific survival (DSS) in patients with CTLA4-High, 70.5\%, vs CTLA4-Low, 43.2\%; $p=0.017$ et al. found a significant association between the presence of abundant levels of TILs, and $\mathrm{CD} 4^{+}, \mathrm{CD} 8^{+}$, CTLA- $4^{+}$stromal $\mathrm{T}$ cells, and $\mathrm{pCR}$ in 33 patients with locally advanced breast cancer undergoing NAC, suggesting that CTLA- $4^{+}$TILs may play a role in creating a good response to chemotherapy [34].

However, TIM-3, which has been studied as a newly emerging and promising coinhibitory molecule expressed on TILs, was contrarily found to be associated with a worse chemotherapy response $(\mathrm{OR}=$ 0.253 , 95\% CI: $0.066-0.974, p=0.047$ ) as measured

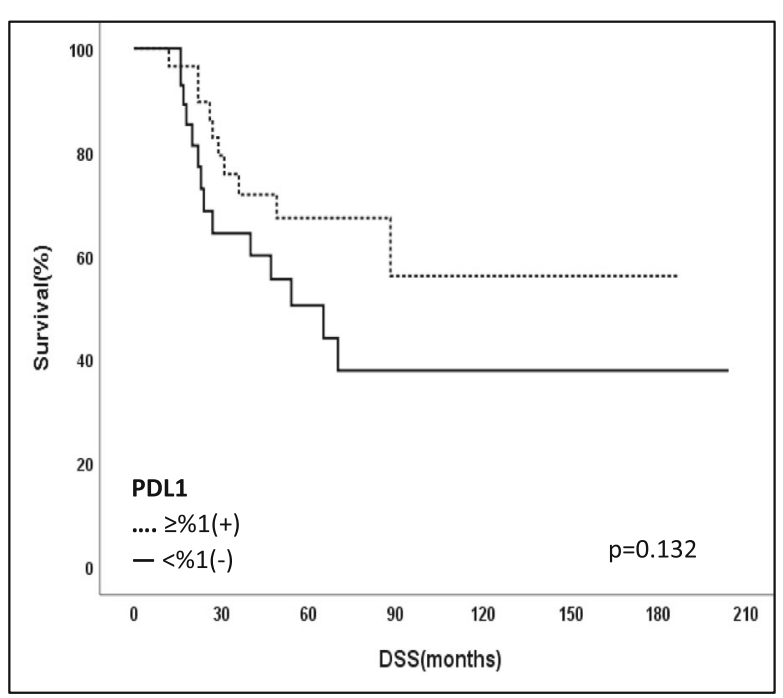

b

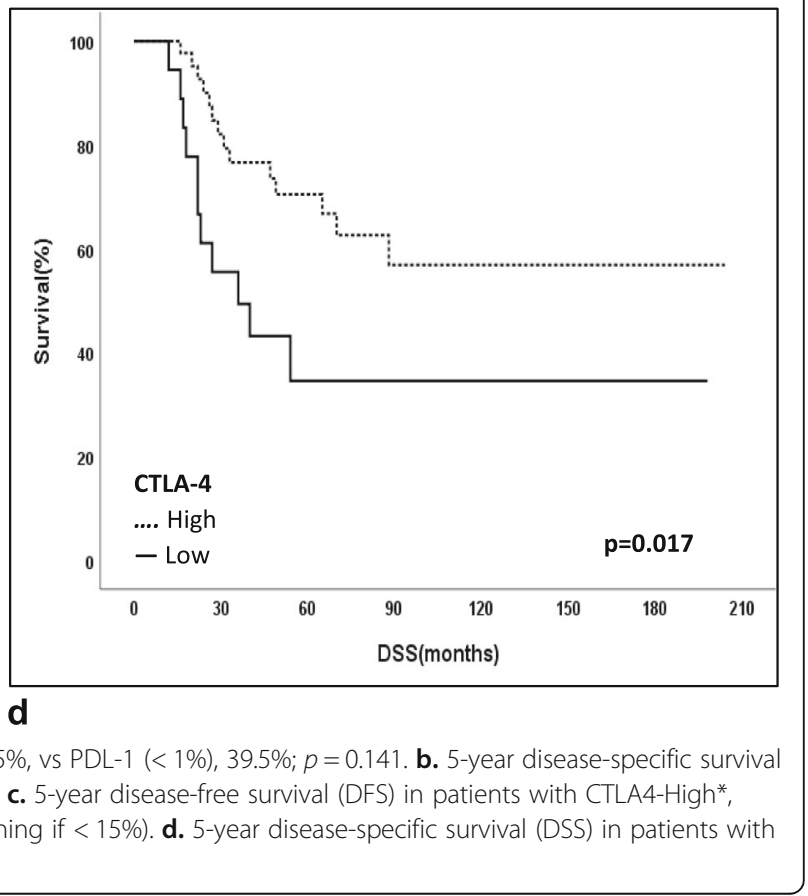

by the MDACC Residual Cancer Burden Index in our study. Using an anti-TIM-3 antibody increased the response to paclitaxel chemotherapy in MMTVPyMT transgenic mice as studied in models of triple-negative and luminal B disease, with no evidence of toxicity [35]. Yashinka et al. further suggested that the TIM-3-galectin-9 pathway may be involved in the immune escape of cancer cells [36]. They reported higher expression of both galectin- 9 and TIM-3 in breast cancer tissues compared to healthy breast tissues of the same patients by 
Table 5 Multivariate Cox Regression Analysis

\begin{tabular}{|c|c|c|c|c|}
\hline & $\begin{array}{l}\text { Disease Free } \\
\text { Survival }\end{array}$ & & $\begin{array}{l}\text { Disease Specific } \\
\text { Survival }\end{array}$ & \\
\hline Factors & HR $(95 \% \mathrm{Cl})$ & $P$ value & HR $(95 \% \mathrm{Cl})$ & $P$ value \\
\hline ypN & & 0.02 & & 0.005 \\
\hline NO & $0.31(0.12-0.83)$ & & $0.21(0.07-0.62)$ & \\
\hline N1-3 & Reference [1] & & Reference [1] & \\
\hline CTLA-4 & & 0.019 & & 0.01 \\
\hline Low & Reference [1] & & Reference [1] & \\
\hline $\mathrm{High}^{\mathrm{a}}$ & $0.38(0.17-0.85)$ & & $0.34(0.15-0.78)$ & \\
\hline
\end{tabular}

Cox Regression (Step-2, Method = Forward Stepwise)

Variables not in the Equation: PDL-1(<\%1 vs $\geq \% 1)$, MDACC Residual Cancer Burden Index (Class 0 vs Class I-III)

Hazard ratio (HR) are presented with their $95 \%$ confidence interval $(\mathrm{Cl})$ and the $\mathrm{p}$-value

a $\geq 15 \%$ \& any moderate/strong staining if $<15 \%$

demonstrating the colocalization of these proteins in breast tumors. Furthermore, Burugu et al. recently investigated the immunohistochemical expression of TIM-3 in 3992 breast cancer specimens in tissue microarray along with other biomarkers, including CD8, PD-1, PD-L1, and LAG-3 [37]. In multivariate analysis, the expression of TIM-3, PD-1, or LAG-3 on TILs was found to be an independent favorable prognostic factor among ER-negative patients. Similarly, Byun et al. reported a better prognosis in patients with TIM-3 expression on TILs in TNBC [38]. These studies also demonstrated that the presence of TIM-3 on TILs was significantly associated with the expression of other ICRs, including PD-1 and LAG-3 on TILs and PD-L1 on tumors. Our findings have shown that the majority of patients (69\%) had TIM3 positivity, and similarly indicated significant correlations between TIM-3 expression on TILs and LAG-3 or PD-1 or PD-L1 on TILs. Even though TIM-3 expression was negatively correlated with response to NAC in our study, we could not demonstrate any prognostic significance of these ICRs in our cohort of patients with TNBC following NAC presenting with more advanced disease compared to other studies. The prognostic significance of TIM-3 expression should be therefore studied in cases with TNBC including different stages in future.

Another novel coinhibitory receptor, LAG-3, which negatively regulates $\mathrm{T}$-cell activation, has been reported to be present at a lower expression rate (18\%) in early-stage TNBC as compared to PD-1 expression on TILs (30\%), whereas coexpression of both receptors was found in $15.4 \%$ of breast-tumorassociated TILs [39]. Similarly, expressions of PD-1 and LAG-3 and concurrent expressions of PD-1 and
LAG-3 on TILs were shown to be 62,26 , and $23 \%$, respectively, in our study. However, the biological significance of the concurrent expression of inhibitory receptors such as PD-1 and LAG-3 is unknown. No prognostic significance could be found in patients with LAG-3 expression alone or concurrent LAG-3 and PD-1 expression in the study of Bottai et al. in concordance with our study. However, a recent meta-analysis of 15 studies including different cancers indicated that LAG-3 expression was associated with better $\mathrm{OS}(\mathrm{HR}=0.81,95 \% \mathrm{CI}: 0.66-0.99$, $p=0.04)$, with a greater magnitude in early-stage malignancies (HR $=0.73,95 \% \mathrm{CI}: 0.60-0.88$ ) and an improved DFS in breast cancer in a subgroup analysis $(\mathrm{HR}=0.64,95 \% \mathrm{CI}: 0.42-0.98)$ [40]. Finally, Burugu et al. [41] studied LAG-3 expression in 4322 breast cancer specimens by immunohistochemistry and reported an improved outcome in patients with LAG-3 expression on TILs with a breast-cancerspecific survival $(\mathrm{HR}=0.71,95 \% \mathrm{CI}$ : 0.56-0.90), particularly among estrogen receptor-negative patients $(\mathrm{HR}=0.50$, 95\% CI: 0.36-0.69). Furthermore, concurrent LAG-3 expression with PDL1 and PD1 was 53 and $61 \%$, respectively. These values seem to be higher than our findings and Bottai's study [39], which might be due to the different clinicopathological characteristics and stages of patients in the study cohorts.

We recently have studied the expressions of immune checkpoints receptors including PD-1, LAG-3, TIM-3, TIGIT and CTLA-4 on CD8 T lymphocytes and Natural Killer (NK) cell subsets obtained from TILs of patients with LABC following NAC by flowcytometry [42]. Our results suggested that HER2+ patients were more likely to have increased TIM-3 expressions on cytotoxic CD8- T cells, and patients with a younger age and advanced tumor burden are more likely to express ICRs on TILs compared to others. More studies are warranted to understand the precise role of LAG-3 or TIM-3 coexpressions on TILs by using different techniques including flow-cytometry or multiplex immunohistochemistry stainings in larger cohorts of TNBC with different stages.

\section{Conclusion}

Our findings indicate that novel immunological biomarkers, including TIM-3 and LAG-3, were highly expressed in TNBC after NAC similar to CTLA-4, PD-1, and PD-L1 showing different predictive and prognostic features. Based on these high expression patterns, combined immune checkpoint inhibitor therapies via CTLA-4 and/or PD-1 or PDL-1 along with TIM-3 or LAG-3 are to be investigated in 


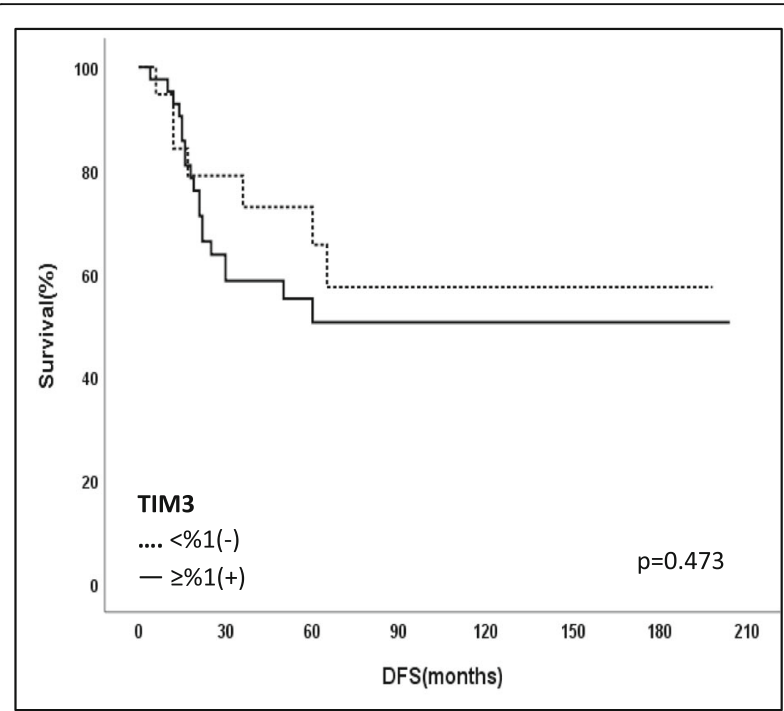

a

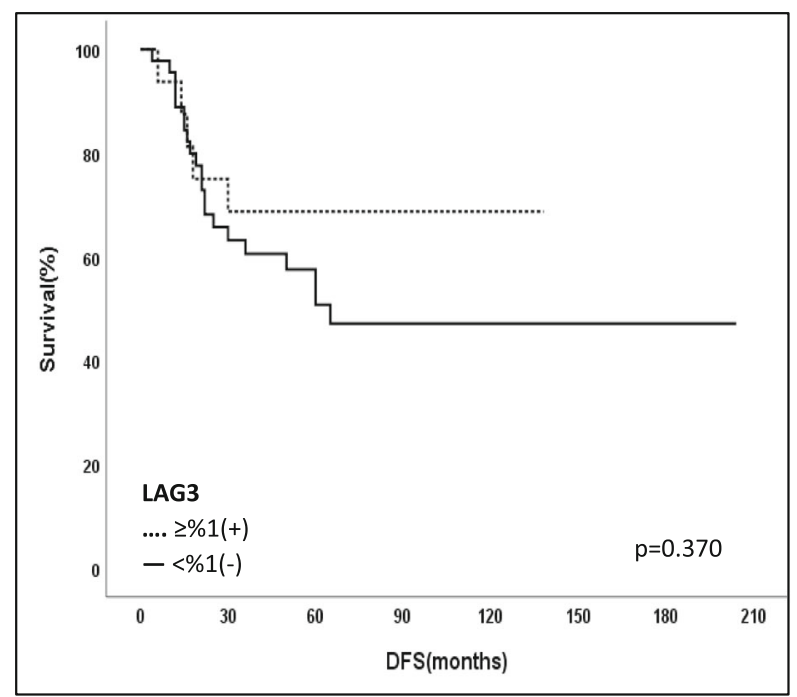

C

Fig. 4 a. 5-year disease-free survival (DFS) in patients with TIM3 $(\geq 1 \%), 50.5 \%$, vs TIM3 $(<1 \%), 65.6 \% ; p=0.473$. b. 5 -year disease-specific survival (DSS) in patients with TIM3 ( $\geq 1 \%)$, 53.6\%, vs TIM3 $(<1 \%), 72.3 \%$; $p=0.524$. c. 5 -year disease-free survival (DFS) in patients with LAG3 ( $\geq 1 \%), 68.8 \%$ vs LAG3 (<1\%), 50.8\%; $p=0.37$. d. 5 -year disease-specific survival (DSS) in patients with LAG3 ( $\geq 1 \%), 60.6 \%$, vs LAG3 $(<1 \%), 59.4 \% ; p=0.778$

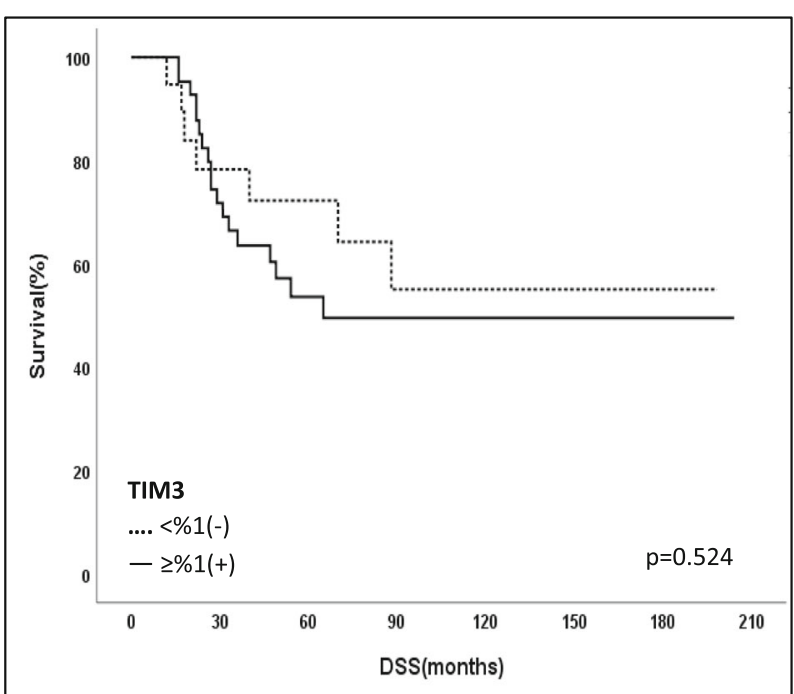

b

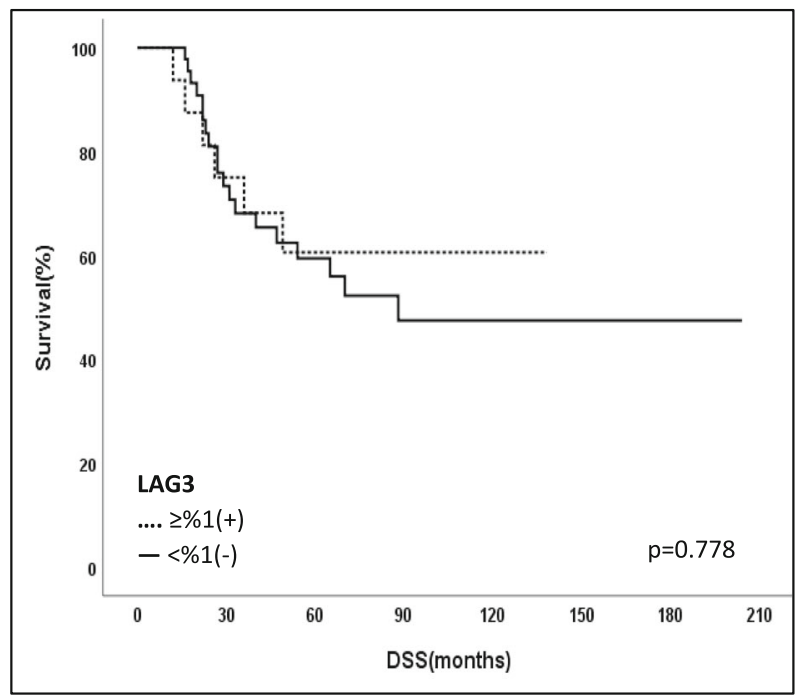

\section{d}

future studies, which may improve the prognosis and pathologic complete response rates of patients undergoing chemotherapy [6, 9-12, 14, 43, 44].

\section{Abbreviations}

ICR: Immune checkpoint receptors; TILs: Tumor-infiltrating lymphocytes; TNBC: Triple-negative breast cancer; NAC: Neoadjuvant chemotherapy; CTLA4: Cytotoxic T Lymphocyte antigen 4; PD-1: Programmed Cell Death 1; PDL1: Programmed Cell Death Ligand 1; TIM-3: T cell immunoglobulin and mucin domain-containing protein 3; LAG-3: Lymphocyte-activation gene 3; FDA: The Food and Drug Administration; ER: Estrogen receptor; PR: Progesterone receptor; HER-2: Human epidermal growth factor receptor2/neu; LABC: Locally advanced breast cancer; pCR: Pathologic complete response; MDACC: MD Anderson Cancer Center; SPSS: Statistical Package for Social Sciences; DFS: Disease-free survival; DSS: Disease-specific survival

\section{Acknowledgements}

The authors thank Fatma Yilmaz and Julide Hocaoğlu for their meticulous technical assistance in immunohistochemical staining, and thank Atilla Bozdoğan for providing statistical analysis.

\section{Authors' contributions}

Acquisition of data (acquired, and managed patient information, and samples): NC, SO, GO, HK, EY. Evaluated IHC: SO, HK. Analysis, interpretation and preparation of data: NC, SO, GO, HK, EY. Accrued patients to study: NC, MT, MM, Al, YE, AA, PS, VO. Writing of the manuscript: NC, SO, GO. Study concept or design: NC. Review and/or revision of the manuscript: MT, MM, $A I, Y E, A A, P S, E Y, V O$. All authors read and approved the final submitted manuscript.

\section{Funding}

This study is funded by the Istanbul University, Department of Scientific Research Projects with the following grant numbers "TTU-2017-26409" and 
"TOA-2017-27023", and by the Istanbul Breast Society. The funding bodies played no role in the design of the study and collection, analysis, and interpretation of data and in writing the manuscript.

\section{Availability of data and materials}

The datasets during and/or analysed during the current study available from the corresponding author on reasonable request.

\section{Declarations}

\section{Ethics approval and consent to participate}

The study was approved by the ethical committee of Istanbul University, Istanbul Medical Faculty. in accordance with the ethical standards of the institutional and/or national research committee and with the 1964 Helsinki declaration. A written informed consent was obtained from all individual participants included in the study.

\section{Consent for publication}

Not applicable.

\section{Competing interests}

The following authors "Neslihan Cabioglu, Semen Onder, Gizem Oner, Hüseyin Karatay, Mustafa Tukenmez, Mahmut Muslumanoglu, Abdullah Igci, Yeşim Eralp, Adnan Aydiner, Pınar Saip, Ekrem Yavuz, Vahit Ozmen have no conflict of interest.

\section{Author details}

'Department of General Surgery, Istanbul Medical Faculty, Breast Surgery Service, Istanbul University, Istanbul, Turkey. ${ }^{2}$ Department of Pathology, Istanbul Medical Faculty, Istanbul University, Istanbul, Turkey. ${ }^{3}$ Current Address: Multidisciplinary Oncologic Centre Antwerp (MOCA), Antwerp University Hospital, Edegem, Belgium. ${ }^{4}$ Current Address: Center for Oncological Research (CORE), University of Antwerp, Wilrijk, Belgium. ${ }^{5}$ Department of Medical Oncology, Institute of Oncology, Istanbul University, Istanbul, Turkey. ${ }^{6}$ Acibadem Maslak Hospital, Acibadem Health Group, Istanbul, Turkey.

\section{Received: 22 October 2020 Accepted: 16 March 2021}

\section{Published online: 06 April 2021}

\section{References}

1. Gajewski TF, Schreiber H, Fu YX. Innate and adaptive immune cells in the tumor microenvironment. Nat Immunol. 2013;14(10):1014-22. https://doi. org/10.1038/ni.2703.

2. Bindea G, Mlecnik B, Tosolini M, Kirilovsky A, Waldner M, Obenauf AC, et al. Spatiotemporal dynamics of intratumoral immune cells reveal the immune landscape in human cancer. Immunity. 2013;39(4):782-95. https://doi.org/1 0.1016/j.immuni.2013.10.003.

3. Nishino M, Ramaiya NH, Hatabu H, Hodi FS. Monitoring immune-checkpoint blockade: response evaluation and biomarker development. Nat Rev Clin Oncol. 2017;14(11):655-68. https://doi.org/10.1038/nrclinonc.2017.88.

4. Kluger HM, Zito CR, Turcu G, Baine MK, Zhang H, Adeniran A, et al. PD-L1 studies across tumor types, its differential expression and predictive value in patients treated with immune checkpoint inhibitors. Clin Cancer Res. 2017; 23(15):4270-9. https://doi.org/10.1158/1078-0432.CCR-16-3146.

5. Reck M, Rodríguez-Abreu D, Robinson AG, Hui R, Csőszi T, Fülöp A, et al. KEYNOTE-024 Investigators. Pembrolizumab versus chemotherapy for PD-L1positive non-small-cell lung cancer. N Engl J Med. 2016;375(19):1823-33. https://doi.org/10.1056/NEJMoa1606774.

6. Larkin J, Chiarion-Sileni V, Gonzales R, Grob JJ, Cowey CL, Lao CD, et al. Combined nivolumab and ipilimumab or monotherapy in untreated melanoma. N Engl J Med. 2015;373(1):23-34. https://doi.org/10.1056/ NEJMoa1504030.

7. Hodi FS, O'Day SJ, McDermott DF, Weber RW, Sosman JA, Haanen JB, et al. Improved survival with ipilimumab in patients with metastatic melanoma. N Engl J Med. 2010;363(8):711-23. https://doi.org/10.1056/NEJMoa1003466.

8. Ellis PM, Vella ET, Ung YC. Immune checkpoint inhibitors for patients with advanced non-small-cell lung cancer: a systematic review. Clin Lung Cancer. 2017;18(5):444-59. https://doi.org/10.1016/j.cllc.2017.02.001.

9. Brignone C, Gutierrez M, Mefti F, Brain E, Jarcau R, Cvitkovic F, et al. First line chemoimmunotherapy in metastatic breast carcinoma: combination of paclitaxel and IMP321 (LAG-3lg) enhances immune responses and antitumor activity. J Transl Med. 2010;8:1-11.

10. Andrews LP, Marciscano AE, Drake CG, Vignali DAA. LAG3 (CD223) as a cancer immunotherapy target. Immunol Rev. 2017;276(1):80-96. https://doi. org/10.1111/imr.12519.

11. Dirix L, Triebel F. AIPAC: a phase IIb study of eftilagimod alpha (IMP321 or LAG-3lg) added to weekly paclitaxel in patients with metastatic breast cancer. Future Oncol. 2019;15(17):1963-73. https://doi.org/10.221 7/fon-2018-0807.

12. Marin-Acevedo JA, Dholaria B, Soyano AE, Knutson KL, Chumsri S, Lou Y. Next generation of immune checkpoint therapy in cancer: new developments and challenges. J Hematol Oncol. 2018;11:1-20.

13. Yano $H$, Andrews LP, Workman CJ, Vignali DAA. Intratumoral regulatory $T$ cells: markers, subsets and their impact on anti-tumor immunity. Immunology. 2019;157(3):232-47. https://doi.org/10.1111/imm.13067.

14. Andrews $L P$, Yano $H$, Vignali DAA. Inhibitory receptors and ligands beyond PD-1, PD-L1 and CTLA-4: breakthroughs or backups. Nature Immunol. 2019; 20(11):1425-34. https://doi.org/10.1038/s41590-019-0512-0.

15. Ibrahim E, Al-Gahmi AM, Zeenelin AA, Zekri JM, Elkhodary TR, Gaballa HE, et al. Basal vs. luminal A breast cancer subtypes: a matched case-control study using estrogen receptor, progesterone receptor, and HER-2 as surrogate markers. Med. Oncol. 2009;26:372-8.

16. Denkert C, Loibl S, Noske A, Roller M, Müller BM, Komor M, et al. Tumorassociated lymphocytes as an independent predictor of response to neoadjuvant chemotherapy in breast cancer. J Clin Oncol. 2010;28(1):10513. https://doi.org/10.1200/JCO.2009.23.7370.

17. Costa R, Shah AN, Santa-Maria CA, Cruz MR, Mahalingam D, Carneiro BA, et al. Targeting epidermal growth factor receptor in triple negative breast cancer: new discoveries and practical insights for drug development. Cancer Treat Rev. 2017:53:111-9. https://doi.org/10.1016/..ctrv.2016.12.010.

18. Denkert C, Liedtke C, Tutt A, von Minckwitz G. Molecular alterations in triple- negative breast cancer-the road to new treatment strategies. Lancet. 2017;389(10087):2430-42. https://doi.org/10.1016/S0140-6736(16)32454-0.

19. Gelmon K, Dent R, Mackey JR, Laing K, McLeod D, Verma S. Targeting triplenegative breast cancer: optimising therapeutic outcomes. Ann Oncol. 2012; 23(9):2223-34. https://doi.org/10.1093/annonc/mds067.

20. Huang M, O'Shaughnessy J, Zhao J, Haiderali A, Cortés J, Ramsey SD, et al. Association of Pathologic Complete Response with long-term survival outcomes in triple-negative breast Cancer: a meta-analysis. Cancer Res. 2020;80(24):5427-34. https://doi.org/10.1158/0008-5472.CAN-20-1792.

21. Wang CJ, Xu Y, Lin Y, Zhu HJ, Zhou YD, Mao F, et al. Platinum-based Neoadjuvant chemotherapy for breast Cancer with BRCA mutations: a meta-analysis. Front Oncol. 2020;10:1-10.

22. Oner G, Altintas S, Canturk Z, Tjalma W, Verhoeven Y, Berckelaer CV, et al. Triple-negative breast cancer-role of immunology: a systemic review. Breast J. 2020;26(5):995-9. https://doi.org/10.1111/tbj.13696.

23. Denkert C, von Minckwitz G, Darb-Esfahani S, Lederer B, Heppner BI, Weber $\mathrm{KE}$, et al. Tumour-infiltrating lymphocytes and prognosis in different subtypes of breast cancer: a pooled analysis of 3771 patients treated with neoadjuvant chemotherapy. Lancet Oncol. 2018;19(1):40-50. https://doi. org/10.1016/S1470-2045(17)30904-X.

24. Loi S, Drubay D, Adams S, Pruneri G, Francis PA. Tumor-infiltrating lymphocytes and prognosis: a pooled individual patient analysis of earlystage triple-negative breast cancers. J Clin Oncol. 2019;37(7):559-70. https:// doi.org/10.1200/JCO.18.01010.

25. Schmid P, Adams S, Rugo HS, Schneeweiss A, Barrios $\mathrm{CH}$, Iwata $\mathrm{H}$, et al. Atezolizumab and nab-paclitaxel in advanced triple-negative breast cancer. N Engl J Med. 2018;379(22):2108-21. https:/doi.org/10.1056/NEJMoa1809615.

26. Marinelli D, Mazzotta M, Pizzuti L, Krasniqi E, Gamucci T, Natoli C, et al. Neoadjuvant Immune-Checkpoint Blockade in Triple-Negative Breast Cancer: Current Evidence and Literature-Based Meta-Analysis of Randomized Trials. Cancers (Basel). 2020;12:2497.

27. Peintinger F, Sinn B, Hatzis C, Albarracin C, Downs-Kelly E, Morkowski J, et al. Reproducibility of residual Cancer burden for prognostic assessment of breast Cancer after Neoadjuvant chemotherapy. Mod Pathol. 2015;28(7): 913-20. https://doi.org/10.1038/modpathol.2015.53.

28. Sharma P, Hu-Lieskovan S, Wargo JA, Ribas A. Primary adaptive and acquired resistance to cancer immunotherapy. Cell. 2017;168(4):707-23. https://doi.org/10.1016/j.cell.2017.01.017.

29. Varun SN, Haytham ES, Rowaida ZT, Anne J, Bassam AR, Eyad E. DNA methylation and repressive $\mathrm{H} 3 \mathrm{~K} 9$ and $\mathrm{H} 3 \mathrm{~K} 27$ trimethylation in the promoter 
regions of PD-L1, CTLA-4, TIM-3, LAG-3, TIGIT, and PD-L1 genes in human primary breast cancer. Clin Epigenetics. 2018;10:1-12.

30. Ren X, Wu H, Lu J, Zhang Y, Luo Y, Xu Q, et al. PD-1 protein expression in tumor infiltrated lymphocytes rather than PDL1 in tumor cells predicts survival in triple negative breast cancer. Cancer Biol Ther. 2018;19(5):373-80. https://doi.org/10.1080/15384047.2018.1423919.

31. Huang W, Ran R, Shao B, Li H. Prognostic and clinicopathological value of PD-L1 expression in primary breast cancer: a meta-analysis. Breast Cancer Res Treat. 2019;178:17-33.

32. Contardi E, Palmisano GL, Tazzari PL, Martelli AM, Falà F, Fabbi M, et al. CTLA-4 is constitutively expressed on tumor cells and can trigger apoptosis upon ligand interaction. Int J Cancer. 2005;117(4):538-50. https://doi.org/1 $0.1002 /$ ijc. 21155

33. Yu H, Yang J, Jiao S, Li Y, Zhang W, Wang J. Cytotoxic T lymphocyte antigen 4 expression in human breast cancer: implications for prognosis. Cancer Immunol Immunother. 2015;64(7):853-60. https://doi. org/10.1007/s00262-015-1696-2.

34. Kaewkangsadan V, Verma C, Eremin JM, Cowley G, llyas M, Eremin O. Crucial contributions by $\mathrm{T}$ lymphocytes (effector, regulatory, and checkpoint inhibitor) and cytokines ( $\mathrm{TH} 1, \mathrm{TH} 2$, and $\mathrm{TH} 17$ ) to a pathological complete response induced by Neoadjuvant chemotherapy in women with breast Cancer. J Immunol Res. 2016;2016:1-25. https://doi.org/10.1155/2016/4757405.

35. De Mingo PA, Gardner A, Hiebler S, Soliman H, Rugo HS, Krummel MF, et al. TIM-3 regulates $\mathrm{CD}_{103^{+}}$dendritic cell function and response to chemotherapy in breast cancer. Cancer Cell. 2018;33:60-74.

36. Yasinska IM, Sakhnevych SS, Pavlova L, Selnø ATH, Abeleira AMT, Benlaouer $\mathrm{Q}$, et al. The Tim-3-Galectin-9 pathway and its regulatory mechanisms in human breast Cancer. Front Immunol. 2019;10:1-13.

37. Burugu S, Gao D, Leung S, Chia SK, Nielsen TO. TIM-3 expression in breast cancer. Oncoimmunology. 2018;7:1-9.

38. Byun KD, Hwang HJ, Park KJ, Kim MC, Cho SH, Ju MH, et al. T-cell immunoglobulin Mucin 3 expression on tumor infiltrating lymphocytes as a positive prognosticator in triple-negative breast Cancer. J Breast Cancer. 2018;21 (4):406-14. https://doi.org/10.4048/jbc.2018.21.e61.

39. Bottai G, Raschioni C, Losurdo A, Tommaso LD, Tinterri C, Torrisi R, et al. An immune stratification reveals a subset of PD-1/LAG-3 double positive triplenegative breast cancers. Breast Cancer Res. 2016:18:1-10.

40. Saleh RR, Peinado P, Fuentes-Antra J, Pérez-Segura P, Pandiella A, Amir E, et al. Prognostic value of lymphocyte-activation gene 3 (LAG3) in cancer: a meta-analysis. Front Oncol. 2019;9:1-9.

41. Burugu S, Gao D, Leung S, Chia SK, Nielsen TO. LAG-3 ${ }^{+}$tumor infiltrating lumphocytes in breast cancer: clinical correlates and association with PD-1/ PD-L1+ tumors. Ann Oncol. 2017;28(12):2977-84. https://doi.org/10.1093/a nnonc/mdx557.

42. Abbasov A, Çetin E, Cabioglu N, Mollavelioglu B, Onder S, Emiroglu S, et al. Abstract 947: Differential expressions of immune check point receptors on tumor infiltrating lymphocytes after neo-adjuvant chemotherapy (NAC) in patients with locally advanced breast cancer (LABC). Proceedings: AACR Annual Meeting 2020; April 27-28, 2020 and June 22-24, 2020; Philadelphia, PA. https://doi.org/10.11 58/1538-7445.AM2020-947.

43. Solinas C, Migliori E, De Silva P, Willard-Gallo K. Review: LAG3: the biological processes that motivate targeting this immune checkpoint molecule in human cancer. Cancers. 2019;11:1-16.

44. Rotte A. Combination of CTLA-4 and PD-1 blockers for treatment of cancer. J Exp Clin Cancer Res. 2019:38:1-12.

\section{Publisher's Note}

Springer Nature remains neutral with regard to jurisdictional claims in published maps and institutional affiliations.

Ready to submit your research? Choose BMC and benefit from:

- fast, convenient online submission

- thorough peer review by experienced researchers in your field

- rapid publication on acceptance

- support for research data, including large and complex data types

- gold Open Access which fosters wider collaboration and increased citations

- maximum visibility for your research: over $100 \mathrm{M}$ website views per year

At $\mathrm{BMC}$, research is always in progress.

Learn more biomedcentral.com/submissions 